\title{
Priming of pop-out: I. Role of features
}

\author{
VERA MALJKOVIC and KEN NAKAYAMA \\ Harvard University, Cambridge, Massachusetts
}

\begin{abstract}
We examined a visual search task, in which observers responded to the high-acuity aspect of a popout target (shape of an odd-colored diamond or vernier offset of an odd spatial-frequency patch). Repetition of the attention-driving feature (color or spatial frequency) in this task primes the popout; repetition of the high-acuity aspect (shape, vernier offset) does not. Priming of pop-out is due to a decaying memory trace of the attention-focusing feature laid down with each trial. The trace exerts a diminishing effect over the following five to eight trials ( $\sim 30 \mathrm{sec})$, and its influence over this time is cumulative. Observers cannot willfully overcome the priming, which suggests that it is passive and autonomous. Both target facilitation and distractor inhibition are evident; the former has a greater effect. The phenomenon shows complete binocular transfer.
\end{abstract}

In a typical visual search experiment, the observer's task is to detect the presence of the odd target within a field of distractors. Two well-known effects have been observed. Serial search is the term attributed to the finding that an increasing number of distractors increases the time necessary to find the target, yielding positive slope functions (Sagi \& Julesz, 1985; Treisman \& Gelade, 1980). Parallel search is the term attributed to the finding that the target is detected equally quickly regardless of the number of distractors, yielding flat reaction time versus distractor number functions. In this situation, the target also "pops out"; that is, attention is automatically drawn to the odd item. The flat functions and the presence of "pop-out" were generally assumed to be causally related. Bravo and Nakayama (1992), however, dissociated these two aspects by adding an additional requirement to the task, which tapped into its attention-focusing aspect. They asked observers to respond to the shape of the odd-colored target, not its presence or absence. It was their claim that responding to the shape necessitates the spatial focusing of attention to the odd target. As such, this task examines the characteristics of attention focusing in pop-out. In contrast, the previously used presence versus absence task does not require the focusing of attention and is not informative regarding the deployment of focal attention.

Drawing on theoretical accounts of attentional guidance, Bravo and Nakayama (1992) made a number of experimental predictions. To start, they reviewed two general processes that would be useful for focusing at-

This work was supported by AFOSR Grant F49620-92-J-0016 to K.N. We thank Shinsuke Shimojo for his comments on an earlier version of this manuscript. V. Maljkovic is now in the Department of Brain and Cognitive Sciences, Massachusetts Institute of Technology. Correspondence should be addressed to K. Nakayama, Department of Psychology, Harvard University, 33 Kirkland Street, Cambridge, MA 02138 (e-mail: ken@wjh12.harvard.edu). tention, broadly classifying them as "top-down" and "bottom-up." First, if the target color is known, then its presentation will immediately draw attention to it. Several theories invoke top-down influence in the selection process. Treisman (1988) suggested that one can potentiate, say, a red feature map, and any activity there will uniquely "mark" an attended site. Thus, attention can be drawn with equal efficiency, regardless of the number of distracting elements. Duncan and Humphreys (1989) proposed the formation of a template with the specifications of the desired target (see also Wolfe, Cave, \& Franzel, 1989). However, if the target color changes from trial to trial, this first and more efficient top-down mechanism cannot operate. It is here that bottom-up mechanisms of attentional guidance are required. Two such mechanisms have been proposed-similarity vetoing (Koch \& Ullman, 1985) and texton gradients (Julesz, 1986). Both are very sensitive to the local density of distractors and targets, and if the visual angle of the display is held more or less constant, attentional deployment should be more effective when the number of distractors is increased.

Bravo and Nakayama's (1992) data supported both expectations. When top-down processing was applicable (i.e., when the target color was known), reaction times were constant with increasing numbers of distractors and were faster overall. When only bottom-up processing could be used (i.e., when the target color was unknown), reaction times decreased with increasing numbers of distractors.

Both theories, as well as Bravo and Nakayama's (1992) data, imply that the knowledge of the attention-driving feature changes the mechanism that one uses to perform a search task. Our interest was to study this apparent switch from one set of mechanisms to the other. We discovered that a priming mechanism is responsible for this dramatic reduction of reaction times in visual pop-out tasks. To anticipate, this priming is surprisingly machinelike and passive. It appears to be dependent on a rapidly 
decaying short-term-memory trace, which can summate over approximately five to eight trials.

\section{GENERAL METHOD}

Recall that in Bravo and Nakayama's (1992) paradigm, observers respond to the shape of the odd-colored target. By design, this approach is well suited to examine pop-out because we can disambiguate the effect of color, which is an attention-driving feature, from the effect of shape, which defines the specific response required. Moreover, there are large differences in reaction times between mixed and blocked conditions, so any changes should be easily observed. Therefore, we continue to use their paradigm with minor modifications.

We first describe the method that, with a few exceptions, was common to all experiments. The exceptions to this method are noted where relevant

In all of the experiments, an observer was confronted with a speeded reaction time task and instructed to respond as quickly as possible to the shape of the odd target. With one major exception (see Experiment 6), the elements were red and green diamonds on a black background; the distractors were all of one color, and the target was the other. Each diamond had the left or right side cut off; this was assigned on a random basis. The diamonds were arranged on an imaginary, almost circular ellipse ${ }^{1}$ around a central fixation point, so that they were roughly equidistant from each other.

\section{Stimuli}

The red and green diamonds were close to equiluminant, chosen so that reaction time for each was comparable. Red had a luminance of $1.98 \mathrm{~cd} / \mathrm{m}^{2}$ and CIE coordinates of $.612 / .351$; green had a luminance of $2.08 \mathrm{~cd} / \mathrm{m}^{2}$ and CIE coordinates of $.313 / .553$. The background had a luminance of $.07 \mathrm{~cd} / \mathrm{m}^{2}$. The diamonds were approximately $1.0^{\circ} \times 1.0^{\circ}$, with $.14^{\circ}$ cut off on either the left or right side. They were arranged on the ellipse with a major and minor axis of $10.0^{\circ}$ and $8.1^{\circ}$, respectively. The white fixation point, which was the center of the ellipse and always stayed on the screen, was $.32^{\circ} \times .37^{\circ}$ and had a luminance of $13.2 \mathrm{~cd} / \mathrm{m}^{2}$

\section{Procedure}

The stimulus, target, and two distractors (only in Experiment 1 could there be more than two distractors) stayed on the screen until the observer responded. The blank screen with a fixation point was then presented for an interval of 2-2.5 sec, after which a new stimulus was presented. The position of the target was randomly chosen on each trial between 12 equidistant points on the ellipse. This procedure is represented in Figure 1.

The observers were instructed to fixate throughout the trial and to respond as quickly as possible by pressing a left or a right mouse button as quickly as possible while trying to keep their error rate reasonably low.

\section{Data Analysis}

The reaction time data shown throughout this paper represent means of correct responses. Observations that fell outside three standard deviations for that particular condition were discarded (around 1\%). Error bars represent standard error of the mean and are shown in the figures for each point, or, where only a single value is shown, this value is a mean of standard errors for all the conditions.

\section{EXPERIMENT 1 Replication of Bravo and Nakayama (1992)}

Bravo and Nakayama used a rectangular stimulus array. Given that we wanted to use more or less equidistant tar-

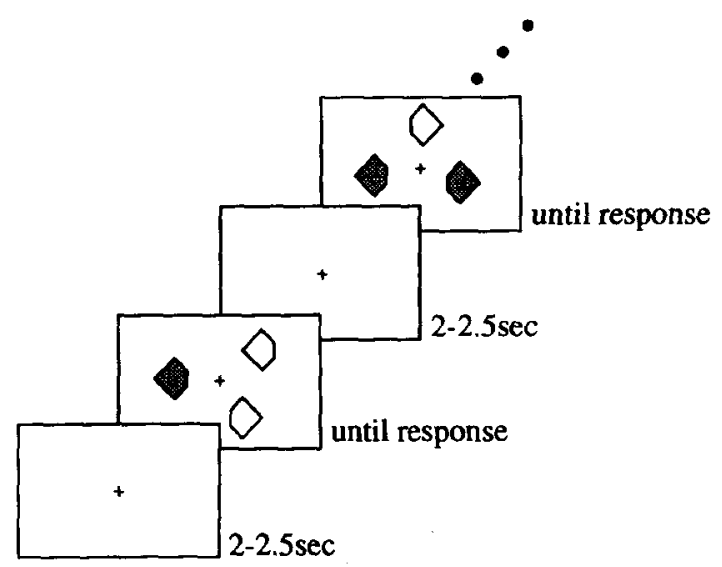

Figure 1. Schematic representation of the stimulus and trial sequence most commonly used in the present paper. The observer's task was to quickly determine whether the odd-colored diamond was cut off on the right or left. (Stimulus is not drawn to scale.)

get elements and also to randomize position (and thus use a roughly circular array), we needed to replicate Bravo and Nakayama's finding with this different spatial configuration.

\section{Method}

In this experiment, the number of distractors could be 2, 3, 5, or 11 . In all other aspects, the method was the same as that described in the General Method.

Subjects. Two naive observers participated in this experiment.

Procedure. The observers participated in two conditions: the "blocked" condition in which the target was always the same color within a block, and the "mixed"2 condition, in which the target and distractor colors were switched unpredictably from trial to trial. The observers' task was to find the odd-colored diamond and to designate which side of this diamond was cut off. The number of distractors and the position of the target varied randomly from trial to trial: intertrial interval was $1,000 \mathrm{msec}$. If an observer made a mistake on a given trial, that trial was repeated at the end of the set.

Both of the observers participated in 320 correct trials in the blocked condition and 320 correct trials in the mixed condition. Trials were presented in four sets of 80 trials, with 4 practice trials before each set. The number of trials for different responses (left/right), target colors (red/green), and number of distractors $(2 / 3 / 5 / 11)$ was equal, and the trials were presented randomly. In the blocked condition, two sets had only red and two sets had only green targets. The sets of "known" and "unknown" targets were interleaved.

\section{Results and Discussion}

As is shown in Figure 2, both of the observers replicated all three findings reported by Bravo and Nakayama (1992). First, the curves are clearly separated in terms of absolute reaction times-mixed-condition times are higher for all distractor-set sizes. Second, the function for the blocked condition is flat, showing no difference in reaction time for increasing distractor number. Third, reaction times fall with increasing distractor number in the mixed condition.

The largest difference between the two conditions is for the two-distractor case. Observers S.S. and E.F.B. 


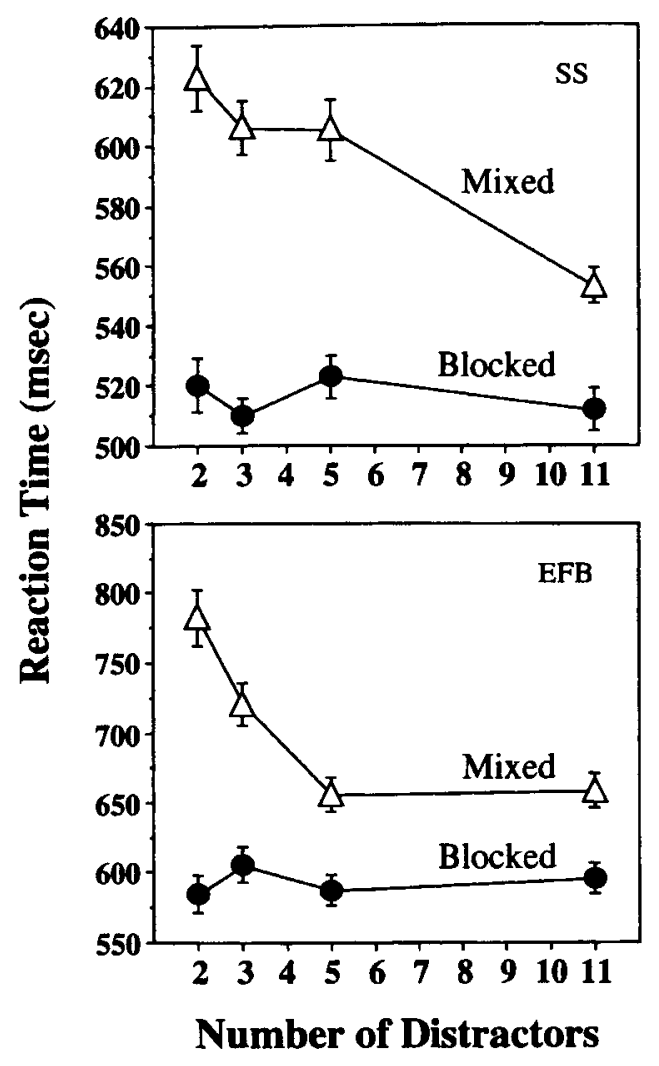

Figure 2. Experiment 1. Relation showing reaction time versus distractor number (replication of Bravo \& Nakayama, 1992). Reaction times decreased with the increasing distractor number when the target and distractor colors changed unpredictably from trial to trial (mixed condition); reaction times remained constant when the colors did not change within a session (blocked condition). Note that reaction times for the blocked condition were generally faster. Error bars in this and all subsequent figures denote standard error of the mean.

show 100-msec and 200-msec differences, respectively, which is typical of the differences found for many observers. (The overall error rates for both of the observers were below $3 \%$.) Because the difference between the blocked and mixed conditions was greatest at this distractor number, we felt that it provided the best opportunity to characterize what was responsible for the much better performance in the blocked condition. Thus, in all of the following experiments, we used only the twodistractor case.

\section{TWO HYPOTHESES}

In order to account for the large difference between the blocked and mixed conditions, we consider two hypotheses.

\section{Stimulus Expectancy}

The most apparent difference between "known" and "unknown" conditions is the uncertainty of the upcoming stimulus. Many studies have shown that target un- certainty leads to deteriorating performance (Cohn \& Wardlaw, 1985; Davis \& Graham, 1981). It is possible that an observer's expectancy of a target color may be responsible for the very large difference in performance seen here. Thus, the stimulus-expectancy hypothesis asserts that if an observer can correctly anticipate the upcoming target color, processing of this color will be more efficient.

\section{Short-Term Memory}

Another very important difference between the blocked and mixed conditions is that, in the former, all the trials in a set had the same target color, and in the latter, the target colors varied from trial to trial. Thus, each will have a very different number of "same-color" trial sequences. In the blocked condition, the probability that the next trial will have the same color is 1.0. In the mixed condition, this probability is .5. Suppose that performance is simply enhanced if the same color appears in consecutive trials - that is, performance is "primed" by the memory of the color on the preceding trial. Such a short-term-memory hypothesis asserts that processing will be more efficient for conditions in which trials are preceded more frequently by the same-color targetsthe extreme being the blocked condition.

The major goal of this paper is to provide evidence to support the priming hypothesis and to detail its characteristics. First, however, we must dispose of the expectancy hypothesis.

\section{EXPERIMENT 2 \\ Pitting Stimulus Expectancy Against Priming}

If expectancy is indeed the critical factor, then it should be possible to manipulate it and see whether it has the predicted effect on performance. It is possible, for example, to keep the mean proportion of target colors at .5, yet manipulate transition probabilities so that the next trial is more or less likely to be different. As one increases the probability of a color switch, $p$ (switch), uncertainty rises to a maximum at .5 and then falls according to the well-known, inverted-U, $-\Sigma p_{i} \ln p_{i}$ relation (where $i$ is an index specifying a given different event, and $p$ is the probability that the event will occur; Attneave, 1959). If observers respond best when there is the least amount of uncertainty, reaction times should show a similar inverted-U function, having a peak at $p($ switch $)=.5$.

Alternatively, however, if short-term memory is the critical factor, a very different prediction follows. A short-term-memory hypothesis would suggest that the greater the number of consecutive trials of the same color, the better the performance, because a residue from the previous trial would prime the same-color trials that follow. So, instead of predicting an inverted- $U$ function as $p$ (switch) is increased, we should see a monotonic rise in reaction times because the number of consecutive repetitions of same-color trials will decrease. 


\section{Method}

Subjects. The two authors, K.N. and V.M., and a naive observer, Z.H., participated in this experiment.

Stimuli. The stimuli were identical to those described in the General Method.

Procedure. The independent variable was the probability that the target and distractor colors would switch on the upcoming trial $-p$ (switch).$^{3}$ Observer K.N. participated in 150 trials, and Z.H., in 200 trials, for each of the following probabilities: 0 (the blocked condition), .5 (the mixed condition), .7, .9, and 1.0. Observer V.M. participated in 600 trials each for the probabilities .1, .5 , and $.9,400$ trials for the probability .7 , and 300 trials for the probability 1.0. It is important to note that with the exception of the case where $p$ (switch) $=0$, the base probability of each color over a set of trials remained at .5 for each value of $p$ (switch). That is, there was almost the same number of red and green stimuli in every block (the computer randomly assigned red or green color to the target and left or right response on every trial), and their number was not preset, as it had been in Experiment 1. The probability of left or right response was .5 throughout the experiment.

\section{Results and Discussion}

The dashed line in Figure 3 shows the kind of facilitation that we would expect from differences in predictability; its boundaries trace scaled plots of the $-\Sigma p_{i} \ln p_{i}$ curve. Although the curve rises from $p$ (switch) $=0$ to $p$ (switch $)=$ .5 as predicted by both theories, it does not fall between .5 and 1.0 as predicted from an uncertainty hypothesis.

Instead of becoming faster, all the observers became slower as $p$ (switch) increased. Consider the extreme case, in which $p$ (switch) was 1.0. Here, the colors alternated on every trial so that predictability was maximum, uncertainty was zero. The stimulus-expectancy hypothesis would suggest that this condition would yield the fastest responding. In fact, all the observers were the slowest, in line with the short-term-memory hypothesis. These findings are not due to a speed-accuracy tradeoff, as is clear from the error rates, which also show a small but noticeable increase with the increase of $p$ (switch).

Prior knowledge, or expectancy of the upcoming color, is clearly not the factor responsible for the difference be- tween blocked and mixed conditions. Our data refuted the stimulus-expectancy hypothesis. They are consistent, however, with the short-term-memory hypothesisexistence of a short-term memory that primes consecutive same-color trials. Moreover, the fact that the observers showed impaired performance with higher predictability and were the slowest when the colors alternated suggests that observers may not be able to use their knowledge to help them with the task, and that a high-level top-down mechanism may not be operating here.

\section{EXPERIMENT 3 Previous-Trial Effect}

In Experiment 3, we explicitly compared performance on consecutive same-color trials with performance on consecutive reverse-color trials. We also made this comparison in three somewhat different conditions. The first one, the basic paradigm, corresponded to the two-distractor case in Experiment 1 . In the second condition, the entire display appeared randomly in the left or the right hemifield. A number of studies (e.g., Rizzolati, Riggio, Dascola, \& Umiltà, 1987; Wurtz, Goldberg, \& Robinson, 1980) have shown that attention is deployed faster within the same hemifield than across the vertical midline to the different hemifield. In the third condition, we reduced the color difference between the target and distractors in order to extend this priming effect to visual search tasks that had a less salient pop-out feature. In all three conditions, the target and distractor colors were equally likely to stay the same and to switch.

\section{Method}

Subjects. Three practiced observers participated in this experiment.

Stimuli. The first condition (the basic paradigm) replicated the $p($ switch $)=.5$ from Experiment 2 and was in every respect identical to the condition described in the General Method. The target in this condition was equally likely to be red or green from trial to
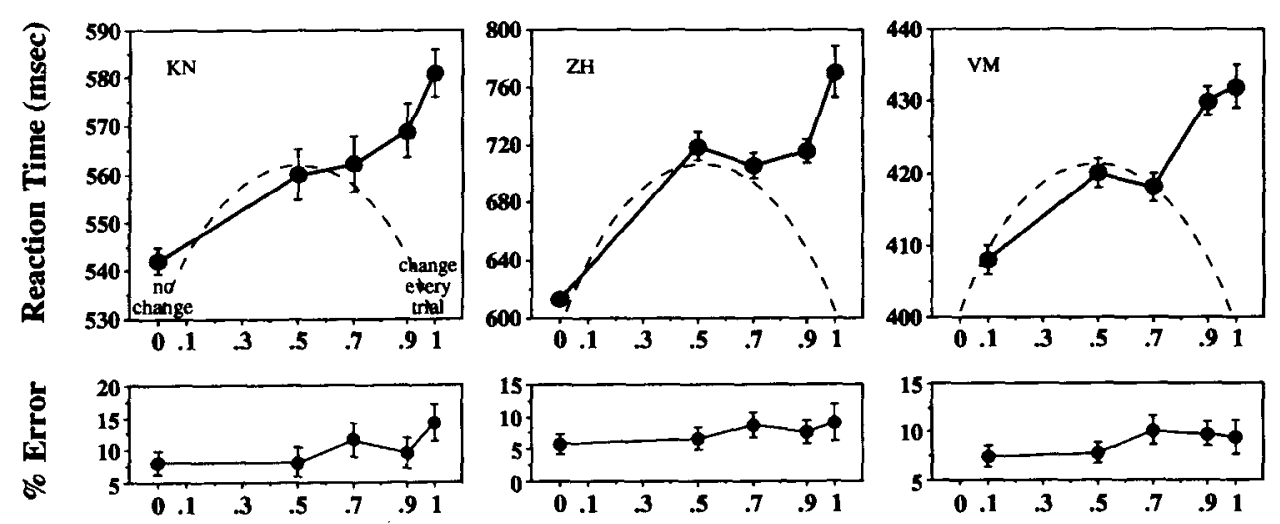

Probability of Color Change

Figure 3. Experiment 2. Relation of reaction time to probability of a color change, $p$ (switch). The "expectancy" hypothesis is represented by the dashed line. Note that with exception of $p($ switch $)=0$, base probabilities for each color are .5. 
trial, was equally likely to appear in any one of 12 positions around the ellipse, and there were always two distractors.

In the second, hemifield, condition, we presented the whole display, target and both distractors, to either the left or right hemifield on a random basis. The target was also equally likely to be red or green from trial to trial. The stimuli used in this condition were larger, approximately $1.25^{\circ} \times 1.50^{\circ}$, with $0.25^{\circ}$ cut off on either the left or right side. They were arranged on an imaginary circle with a diameter of $2.3^{\circ}$, with a center that was $4.5^{\circ}$ away from the fixation point. Elements were equidistant from each other, and the target could fall on any of the 12 positions along the imaginary circle. The center of the display on either side was not marked; only the fixation point, at the center of the screen, was always present.

Finally, in the reduced-color-difference condition, we made the two colors very similar to each other-orange-yellow and ocheryellow instead of the usual red and green. In this condition, the observer participated in four sets of 200 trials each. The orangeyellow had CIE coordinates of .541/.411 and a luminance of $8.17 \mathrm{~cd} / \mathrm{m}^{2}$, and the ocher-yellow had CIE coordinates of $.484 / .453$ and a luminance of $8.62 \mathrm{~cd} / \mathrm{m}^{2}$

Procedure. Observer Z.H. participated in 160 trials of the basic paradigm, K.N. in 180 trials of the basic paradigm and 800 trials of the reduced-color-difference condition, and V.M. in 900 trials of the hemifield condition. All were instructed to fixate throughout the trial and to respond as quickly as possible without making a significant number of errors. In all other aspects, these conditions were identical to those in the General Method.

\section{Results and Discussion}

Figure 4 shows data from the three conditions. In each case, the observer was faster when two consecutive trials were of the same color than when the colors were reversed. The differences ranged from $30 \mathrm{msec}$ to $110 \mathrm{msec}$, depending on the observer. The difference in error rates, where evident, were consistent with a better accuracy for same-color sequences, indicating that the results were not due to a speed-accuracy tradeoff.

Basic paradigm
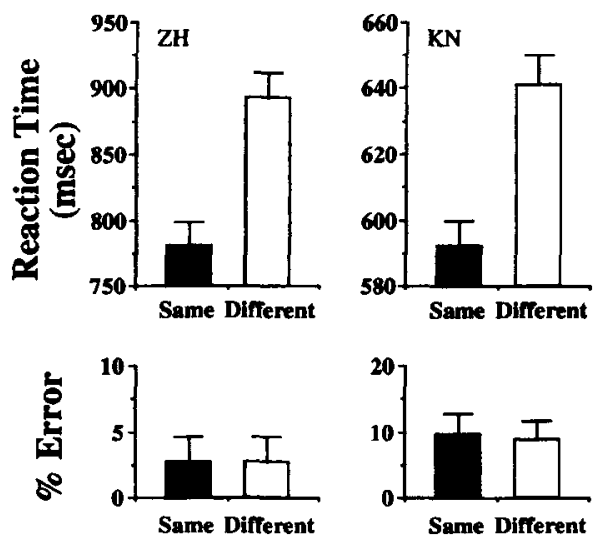

The two variations we performed on the basic paradigm did not produce any noticeable effects. Same color was seen just as quickly in the opposite hemifield as it was in the same hemifield (reaction times are identical: $428 \mathrm{msec}$ for each); different color was seen just as slowly in the same hemifield as it was in the different hemifield (455 msec in the same hemifield; $457 \mathrm{msec}$ in the different hemifield). Reduction of the color difference also did not diminish the effect. The facilitation for the same color on the consecutive trial seems to be quite robust.

Our results indicate a priming for color. But what about shape? So far, we have neglected this dimension because we have assumed that priming occurs only for the dimension that determines pop-out. A high-acuity shape task has been used simply to make sure that spatially focused attention is required. Tacitly, we have assumed that shape is not primed, and we have not varied the contingencies of shape in an analogous manner. Yet, embedded in our data set is the information needed to evaluate this assumption. Throughout the experiments, shape was equally likely to be "left" or "right." If priming for shape is similar to that for color, two same consecutive responses should be faster than two different consecutive responses. ${ }^{4}$ We conducted such an analysis; the results are plotted in Figure 5.

From the data in Figure 5, there is a hint that a tiny amount of shape priming may exist-reaction times are slightly longer for all "previous different" cases. However, error rates in three out of four cases show the opposite trend, suggesting a speed-accuracy tradeoff. Even where errors and reaction times show the same trends, as in the hemifield case, priming of shape/response is clearly dwarfed when compared with color priming (see Figure 4).

\section{Reduced color difference}
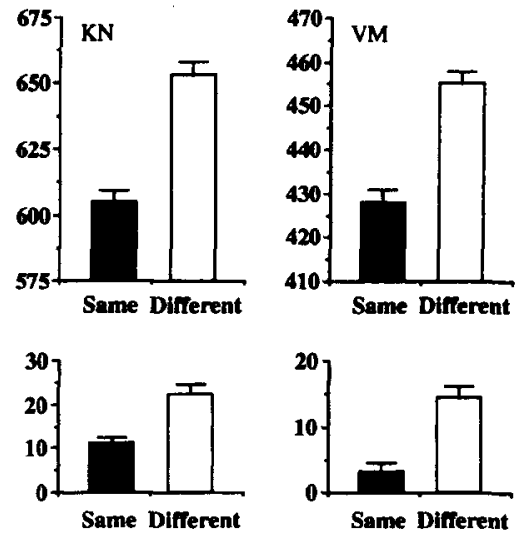

\section{Previous Color}

Figure 4. Experiment 3. Comparison of reaction times and error rates for trials in which the previous color was the same and for those in which the previous color was different. In all three conditions (see text for details), the observers were faster when the color was repeated on two consecutive trials and were slower when it was changed. Error rates show no evidence of a speedaccuracy tradeoff. 


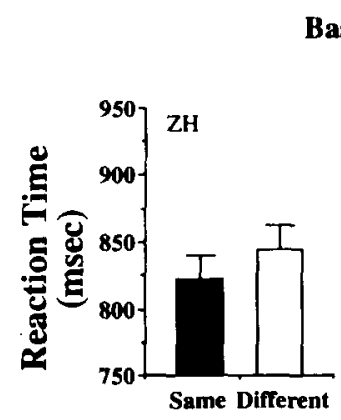

Basic paradigm
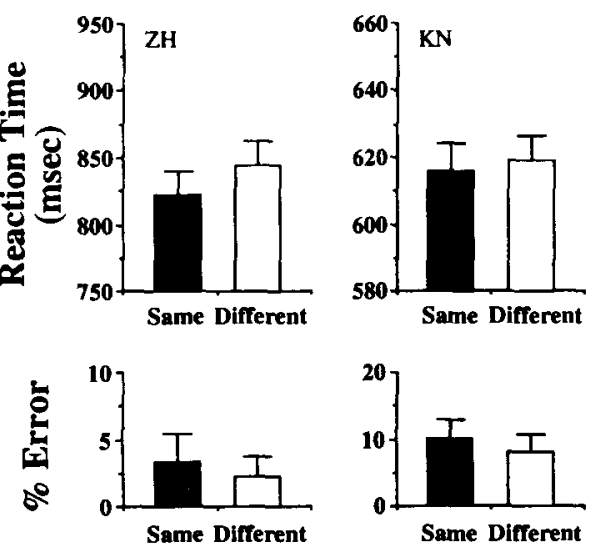

Reduced color difference
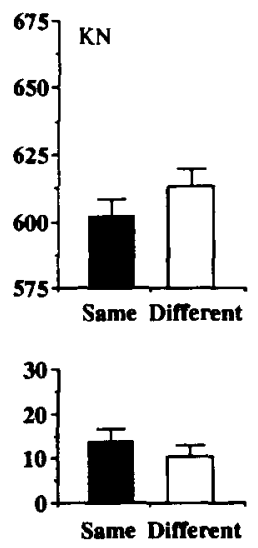

Hemifield
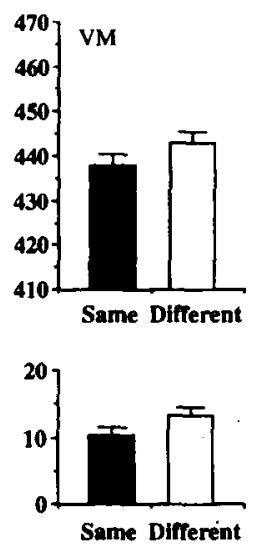

\section{Previous Response}

Figure 5. Experiment 3. A comparison of reaction times and error rates when the shape/response on the previous trial was the same and when it was different. Previous color results from the same set of data are shown in Figure 4. A small decrease in reaction time for the same shape or response is discernible, but note the opposite trend in errors in all but one condition.

\section{EXPERIMENT 4 Lack of Conscious Control}

In this experiment, we asked whether an observer's conscious expectations could influence the amount of priming. In Experiment 2 we showed that when colors alternated, the observers' reaction times were the slowest despite complete predictability of the upcoming color. However, despite the complete predictability, we did not draw observers' attention to this regularity. Observers could have ignored the pattern. In the present experiment, we addressed this issue more exhaustively. Given a completely predictable color sequence as well as an alert observer who is actively trying to take advantage of this predictability, can the observer influence his perceptual processes?

To test this possibility, we employed the following logic. In Experiment 3, we saw that color repetition in a completely unpredictable sequence leads to faster responding. We can think of this facilitation, which apparently happens in a very passive way, as the baseline priming effect. Now we need to compare this passive priming with priming in a condition in which an observer actively attempts to take advantage of predictability. In other words, we need to have conditions in which we can test priming while having complete predictability. In Experiment 2, in the condition in which the colors alternated on every trial, we could look at complete predictability, but not at priming. In this experiment, therefore, we used a double alternating sequence--two red, two green, two red, two green, and so on. This way, we could see how much priming there is with repetition and also have a completely predictable sequence. We also used two modes of doing the task. In the "passive" condition, the observers were instructed to relax and respond to what they saw, but not to put any extra effort into responding. ${ }^{5}$ In the "active" condition, they were instructed to subvocally utter the color that would be in the upcoming trial, ensuring that they were indeed expecting the color that would be presented. The passive condition should provide a baseline priming similar to that observed in Experiment 3. If the observer's active efforts are successful, the amount of priming in the active condition should be less than that in the passive condition. That is, absence or reduction of priming in the active condition would show that this priming phenomenon can be influenced by conscious effort.

\section{Method}

Subjects. Two practiced observers, K.N. and S.S., and a naive observer, G.P., participated in this experiment.

Stimuli. The stimuli were identical to those described in the General Method.

Procedure. We gave the observers sequences of two trials - two red, two green, two red, and so on. We also ran the blocked condition, in which all the trials were of the same color, to establish an additional baseline. The independent measure was the manner in which the observer performed the task - active or passive-and whether the color was presented the first time or was repeated. The dependent measure was the reaction time for correct trials. In all other aspects, the procedure was the same as that described previously. Observers K.N. and G.P. participated in 240 trials for each passive and active condition, and 240 and 160 baseline trials (blocked condition), respectively. Observer S.S. participated in 100 trials for each passive and active condition, and 160 baseline trials.

\section{Results and Discussion}

First, we note the consistently shorter reaction times in the active condition than in the passive condition (see Figure 6). This is not surprising, considering the alert- 

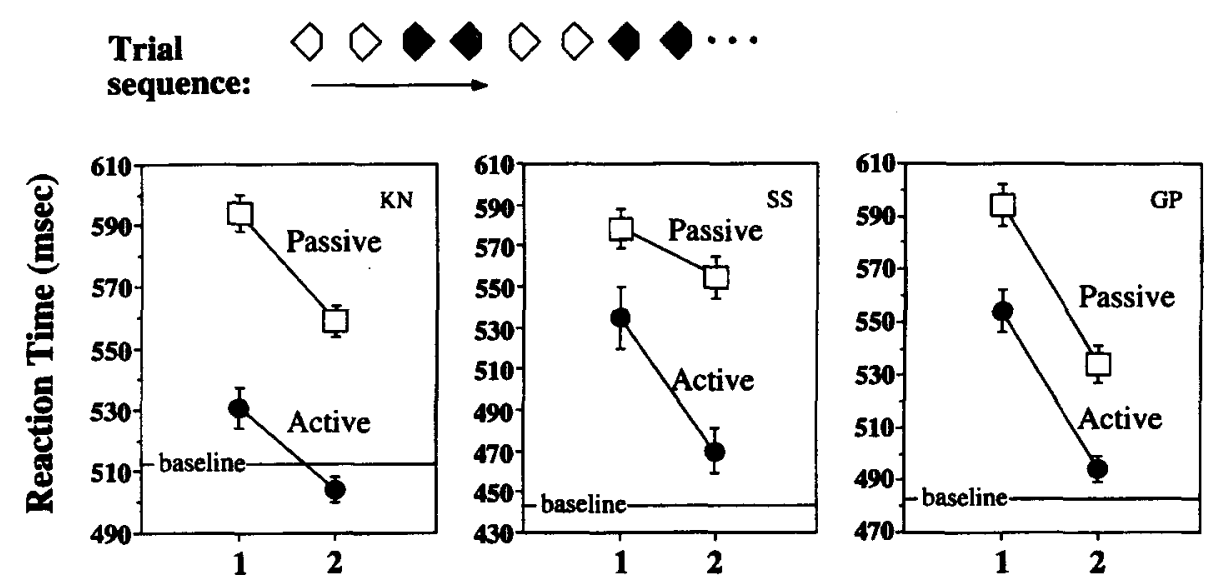

\title{
Order in Sequence
}

\begin{abstract}
Figure 6. Experiment 4. No evidence of conscious influence. Upper inset shows trial sequence in which upcoming colors were completely predictable - a double alternation of two reds, two greens, and so on. In the passive condition, the observers were engaged in other activities in addition to the task. In the active condition, they anticipated each target color subvocally. Graphs show the reaction time as a function of the order in a same-color sequence. Contrary to the conscious-influence hypothesis, there is no diminution in the effect with the repetition of the color in the active condition.
\end{abstract}

ness level required to prepare for the color of each forthcoming trial. More important, however, is the consistent evidence for priming. The difference between the first and second trials in the active condition is not diminished.

Interestingly, Observer S.S. shows an even greater facilitation for the second trial in the active condition than in the passive condition. As mentioned earlier, if this priming phenomenon could be influenced by conscious effort, there should be either no priming or a diminution of priming in the active condition. With this observer we found the opposite-priming was even stronger in the active condition than in the passive condition. These data are therefore contrary to the conscious-influence hypothesis, and they support a passive effect of memory on performance.

\section{EXPERIMENT 5 \\ Influence of a Single Target Exposure Over Time}

In Experiments 3 and 4, we examined only the previoustrial effect, in which we assessed the influence of trial $n-1$. In Experiment 5, we examined the influence of a single trial over a greater time interval. We asked whether the trial previous to the last one could exert an influence on the current trial, whether the trial previous to that could influence the current trial, and so on. In other words we looked at the trial $n-i$, where $i$ can have any value.

\section{Method}

Subjects. Two observers participated in this experiment. K.N. was practiced, and E.F.B. was naive.

Stimuli. For K.N., stimuli identical to those used in the reducedcolor-difference condition in Experiment 4 were used. Observer E.F.B. participated in the basic paradigm, with stimuli that were identical to those described in the General Method.
Procedure. Observer K.N. participated in 1,000 trials of reducedcolor-difference condition-five sets of 200 trials. We pooled these 1,000 trials with the results from his 800 trials in Experiment 3, for a total of 1,800 trials. Observer E.F.B. participated in 2,000 trials of the basic paradigm -10 sets of 200 trials.

\section{Results and Discussion}

In this experiment, we examined whether the color of the stimulus that was $i$ trials back had an influence on the current trial. A stimulus that was $i$ trials back could have a color that was the same as or different from the current stimulus. Note that the influence of stimuli between the trial $n-i$ and $n$ is averaged out (over a large number of trials there will be an equal number of sameand different-color trials for each of the in-between values). We analyzed the effects of 15 trials before the current trial. In addition, we did the same kind of analysis for the trials occurring after the current trial. Obviously, these trials should not exert any influence on the current trials, as they have not occurred yet. They are a good measure, however, of the baseline variability of reaction times. Therefore, we also plotted the "influence" of the seven future trials.

These data are shown in Figure 7. On the $x$-axis is the past/future trial dimension, and on the $y$-axis is reaction time for correct trials. The current trial (the overall mean) is marked by the square. Filled circles are reaction times for preceding trials that had the same color as that in the current trial; open triangles show reaction times for preceding different-color trials. Both of the observers show a large impact of the previous trial on the current trial, replicating the finding from Experiment 3 . Interestingly, the second trial back exerted considerable influence on the current trial by itself, as did the trials as far as five trials back, and possibly further. In order to have a sim- 


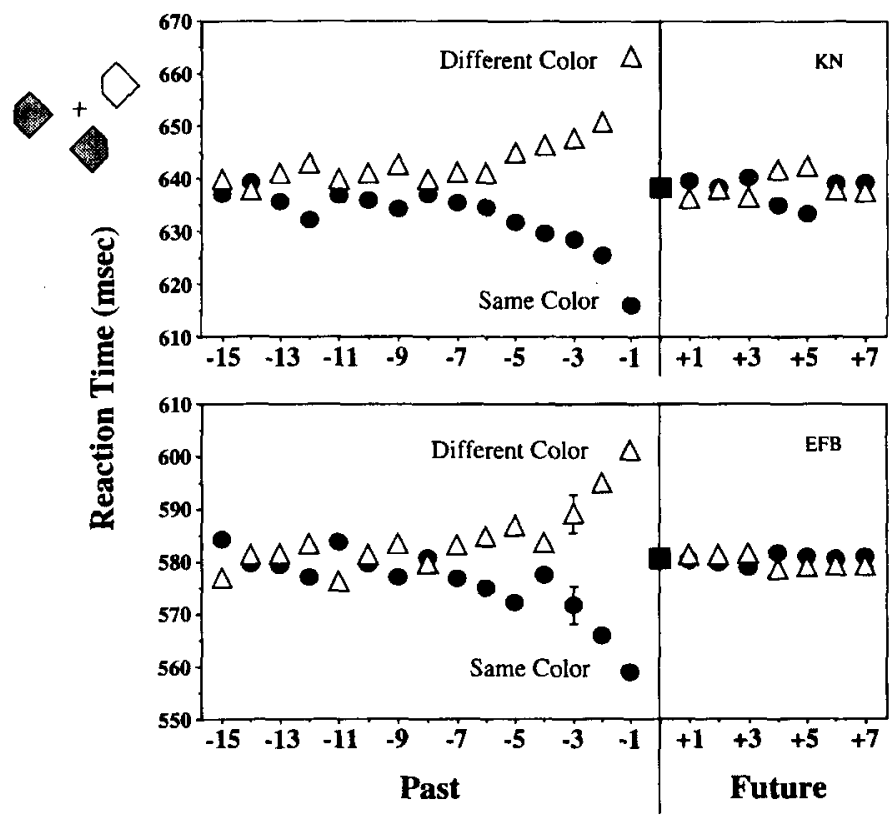

Figure 7. Experiment 5. How does the color of a single trial in the past (and future) influence the current trial? The current trial is marked by a square representing the mean reaction time. For each relative serial position (past and future), reaction time scores are partitioned with respect to whether they had a target color that was the same as that of the current trial (filled circles), or whether they had a different color (open triangles). Standard error bars in the upper panel are smaller than the depicted symbols.

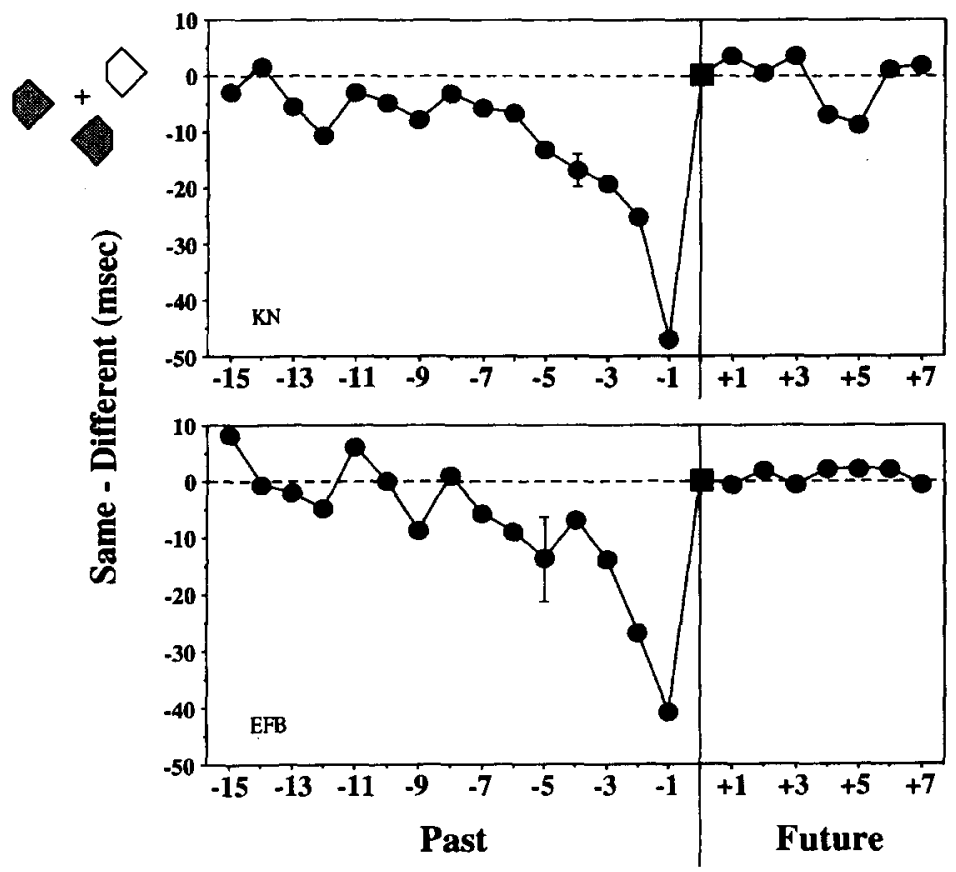

Figure 8. Experiment 5. Same data as those in Figure 7, except here they are plotted as the difference in reaction time between same and different colors. 
pler picture of this facilitation effect, we plotted the same data in another way. We subtracted reaction times for the different-color trials from the reaction times for the same-color trials. This shows how much faster the observers were when a trial that was $i$ trials back was of the same color. These data are shown in Figure 8.

Again, the same conclusion is apparent-a trial was facilitated by the same-color trial at least five trials back. If we think in terms of what happens after the presentation of a single trial, then facilitation is the strongest for the immediately following trial, and it becomes progressively weaker over the following five to eight trials. The facilitation is pronounced-the first consecutive same-color trial is about $45 \mathrm{msec}$ faster than the differentcolor trial.

These data support and extend findings from the previous experiments. Not only is it easier to respond to the upcoming same-color trial, but it is easier to do so five trials after a given trial. It seems highly improbable that, in a completely random sequence, an observer constantly kept the last five or more trials in an explicit working memory and consciously prepared a response that was based on the probabilities of the two colors over those five to eight trials. Jarvik (1951) reported that, on the contrary, observers are much more likely to predict the opposite stimulus after two repetitions in an unpredictable two-choice sequence (the gambler's fallacy). Therefore, the results add strong support for the passivememory process. Beyond this, however, they also detail its general time course, showing that the effect of a sin- gle trial exposure takes approximately five to eight trials to decay.

\section{EXPERIMENT 6 \\ Memory for a Different Attention-Focusing Feature: Spatial Frequency}

In the previous experiments, we used color as the attention-driving feature. In Experiment 6, we wished to generalize our results, showing that it is not color popout alone that can be primed, but pop-out of any attentionfocusing feature. To accomplish this goal, we used the same paradigm-a high-acuity "shape" task on a popout target - but the odd target was defined by spatial frequency, not color. We used Gabor patches, so that the target would have a different spatial frequency than the distractors. The observers' task was to determine whether the upper half of the target was shifted to the left or to the right from the vertical-a vernier task. Bravo and Nakayama (1992) showed that this variant of the task also produces faster reaction times with increased number of distractors, just as does the color task.

\section{Method}

Subjects. Observers V.M. and G.P. participated in this experiment.

Stimuli. The stimuli consisted of Gabor patches, which are spatially localized sine-wave gratings, whose modulation envelope is a two-dimensional Gaussian function. The suprathreshold area of the Gabor patches subtended approximately $1.8^{\circ}$. They were of either high or low spatial frequency $(3.15$ cycles/degree, and

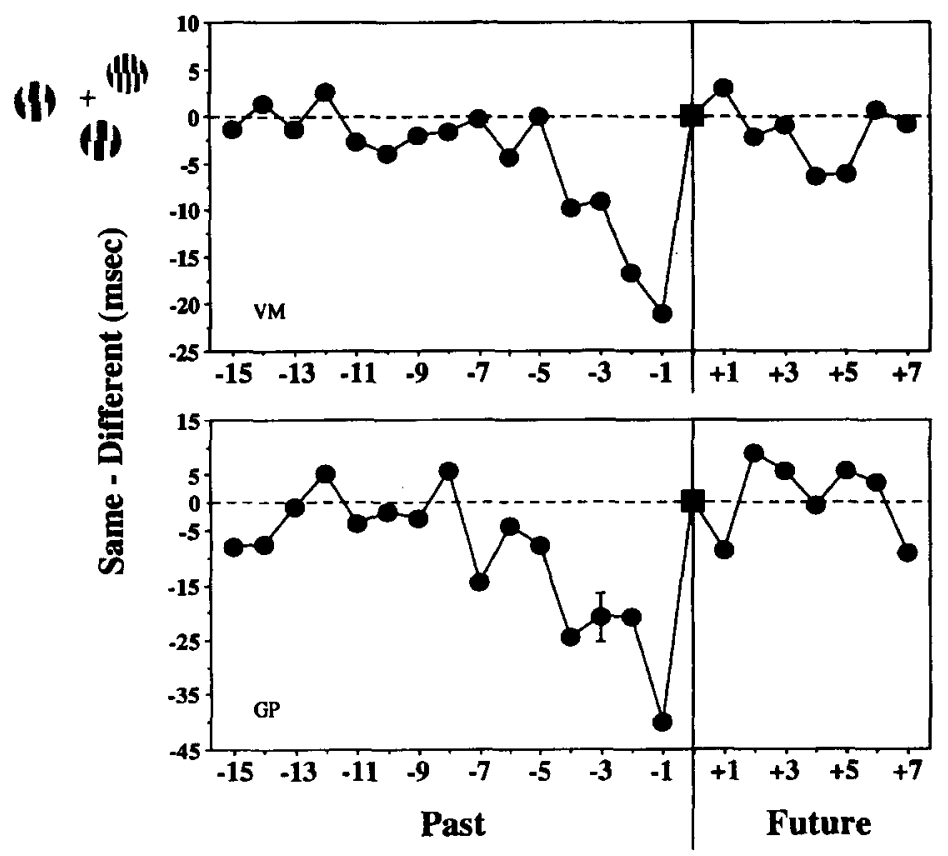

Figure 9. Experiment 6. How does the target spatial frequency of a single trial in the past (and future) influence the current trial? Shown is a replication using spatial frequency as a different attention-focusing feature. Observers again performed a relatively high-acuity task on the pop-out target, judging the vernier offset of an odd spatial-frequency patch (see inset). Difference scores are plotted as a function of relative serial position (as in Figure 8). 
1.66 cycles/degree, respectively) and had a vernier offset of the upper half that was $0.09^{\circ}$ of visual angle to the left or right.

Procedure. The procedure was identical to that described in the General Method. V.M. participated in 3,600 trials in sets of 200; G.P. participated in 2,600 trials in sets of 200 . Due to the large number of trials necessary for this kind of analysis, both of the observers participated on 3 separate days, and then all of the data were combined. We did not observe any significant change in performance over this time, except for slightly faster responding with more practice.

\section{Results and Discussion}

We examined the effect of a single trial in the past. Figure 9 shows how much faster the observers were for the same spatial-frequency target than for the different spatial-frequency target.

It is evident that performance of the vernier task on the same-frequency target was significantly faster than performance on the different-frequency target. This replicates the same basic pattern of results seen for color and supports the hypothesis that priming of pop-out is not specific to color, but occurs for the attention-driving feature in general.

\section{SHAPE/RESPONSE PRIMING}

So far, we have discussed the existence of short-term memory for the attention-focusing feature, its duration over about five to eight trials, and its passive nature (it is not influenced by conscious efforts). In this task, as most of our readers have probably noticed by now, there are two additional variables that may be primed - the position of the stimulus, and its shape. We will address priming of position separately in a forthcoming paper. To preview the results briefly, we find that position priming is remarkably similar to color priming. There is facilitation when target position is repeated on the consecutive trial, and inhibition when a target falls where a distractor used to be. The priming is graded, so that there is slight facilitation for positions adjacent to the target and slight inhibition for positions adjacent to the distractors. The effect of a single trial also lasts for about five to eight trials. Although position priming is remarkably similar to color and spatial-frequency priming, it is independent of it (Maljkovic, Bravo, \& Nakayama, 1992).

We have touched upon the priming of the shape previously, and now we present some more data. We would like to reiterate here that shape and response are confounded in our task, color and other variables are not. Therefore, all of the "shape" data in this paper are at the same time "response" data.

Data from Experiment 3 suggested that priming for the shape was minimal, if any. In order to check and examine in more detail the possible existence of shape/response priming, we performed the single-trial-effect analysis on the response data from Experiments 5 and 6 .

In Figure 10, we show these shape/response data (filled circles) for the color and the spatial-frequency experiments (upper and lower panels, respectively). The dashed lines trace out the data for the attention-focusing feature of the given experiment, which we presented previously; they are shown in the figure for comparison. Note that the variability not due to memory is presented in the "future" trials. These trials had not yet occurred; therefore, they could not have had an effect on the current trial.

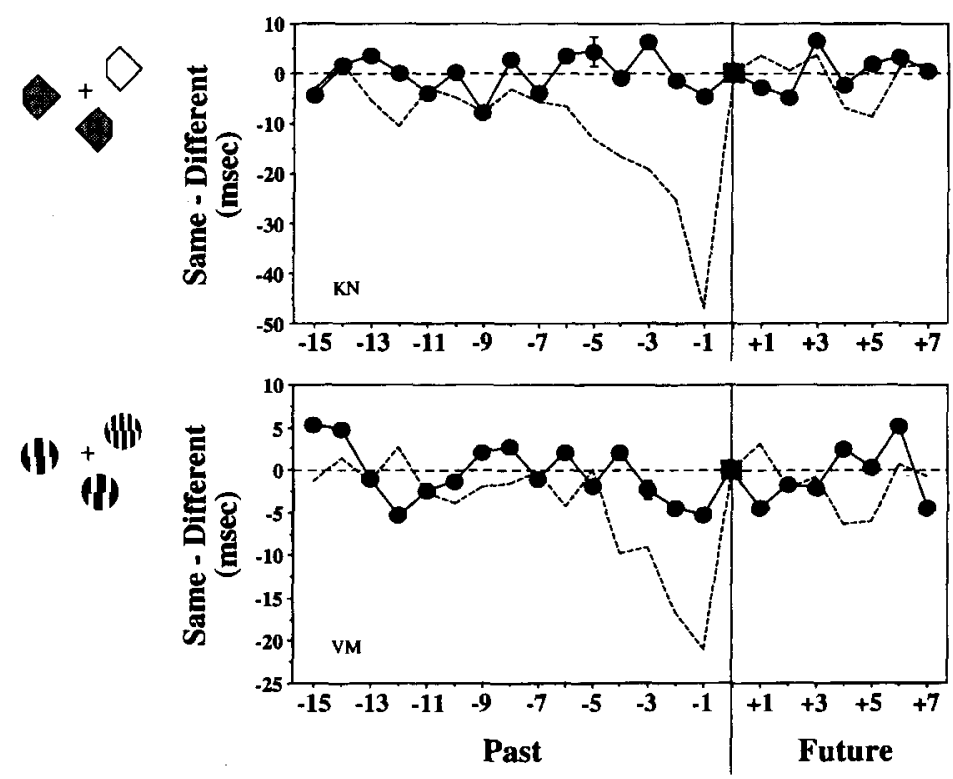

Figure 10. Alternative analysis of Experiments 5 and 6, showing that single responses in the past have little or no systematic influence on a current trial. Filled circles show the difference between same and different responses (calculated as in previous two figures) as a function of relative serial position. For comparison, the dashed line shows differences for color and spatial frequency, respectively. (Note that the appropriate baseline is the variability in the future trials.) 
Any effect of the preceding trials that falls within the variability of the future trials can be said to have occurred by chance. It should thus be apparent from these graphs that response/shape shows very little, if any, priming ${ }^{6}-$ a result very different from that of color or spatial frequency.

\section{EXPERIMENT 7 Cumulative Effects of Priming}

Now that we have established that the memory is formed for the attention-focusing feature in general, we will more carefully examine a consequence of this memory and offer a hypothesis for the solution of the problem we posed at the beginning of the paper. Bravo and Nakayama (1992) found an effect of decreasing reaction times with increasing distractor number only in the mixed condition-that is, when the target and distractor colors were switched unpredictably from trial to trial. Earlier in this paper, we characterized the dramatic facilitation that was seen in the blocked condition when compared with the mixed condition. We showed that it was not the knowledge or the predictability of the color on the upcoming trial, but rather the memory of the color acting for several trials after its appearance that produced the facilitation. Reaction time differences in Bravo and Nakayama's experiment ranged between 100 and $200 \mathrm{msec}$ (for the two-distractor case in the mixed and blocked conditions). If we consider just the previous-trial results obtained in Experiments 3-6, we note that reaction times for the same-color trial are approximately 30 $50 \mathrm{msec}$ shorter than those for the opposite-color trial. This reaction time difference is not sufficient to explain the blocked-mixed difference of over $100 \mathrm{msec}$. Findings from Experiments 5 and 6 suggest a way to reconcile this difference: We can assume that the effects of several trials summate to produce the difference between blocked and mixed conditions. If the facilitation effects indeed sum over trials, we might expect this to happen for up to about eight trials.

In order to efficiently examine the performance during long sequences, ${ }^{7}$ we employed the following strategy. We generated three different lengths of same-color trials, and each sequence had an equal chance of occurring. In this and subsequent experiments, we continued to use the color stimuli.

\section{Method}

Subjects. Practiced observer K.N. and a naive observer, F.P., participated in this experiment. Neither observer was aware of the purpose of the experiment.

Stimuli. The stimuli were identical to those described in the General Method.

Procedure. The procedure differed slightly for the 2 observers. Observer F.P. had sequence lengths of 2, 6, and 10, and these sequences came up randomly. Observer K.N. had sequences of 4,8 , and 11 . This observer participated in two different conditionsone in which he ran blocks of trials with sequence lengths that were always the same (always 4,8 , or 11 ), and one in which he ran interleaved sequences of $2,4,6,8$, and 11 . These conditions changed randomly. The observers were not told that the sequence lengths were preset, and when they were queried afterward, they claimed no awareness that the trials were anything other than randomly presented.

\section{Results and Discussion}

The results from this experiment are shown in Figure 11. On the $x$-axis, we plotted the order in which a trial appeared within its sequence (whether the trial was the first, second, third, etc. time that a given color was seen). Reaction time for correct trials is on the $y$-axis. The baseline shows reaction times in blocked conditions for each of these observers.

Both of the observers showed a gradual decrease in reaction times with each color repetition. Moreover, by the eighth trial in the sequence, they reached the speed of the blocked condition, as might be expected from data obtained in Experiments 5 and 6 . As such, the data support the view that the effects of consecutive same-color trials summate. This cumulative priming by only five to eight same-color trials accounts for the large difference between the mixed and blocked conditions in Bravo and Nakayama (1992).

From Experiments 5 through 7, we can estimate the time over which the memory trace can have an effect. A

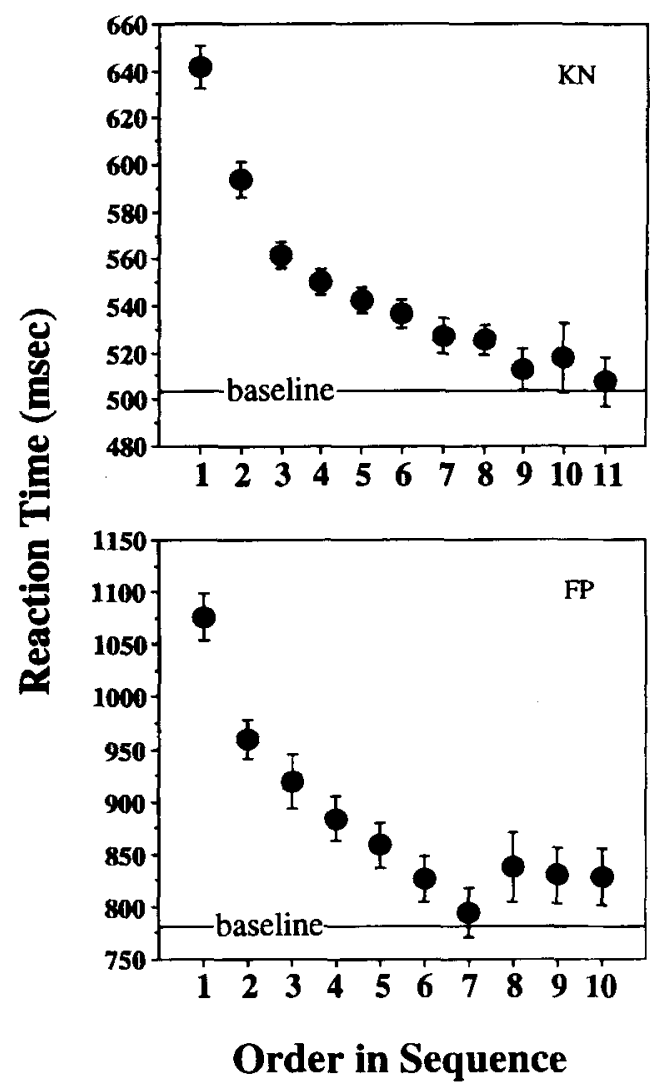

Figure 11. Experiment 7. Decrease in reaction times for successive presentations in a same-color target sequence. The order in which a stimulus appeared within a sequence of same-color trials (whether this was the first red trial in the sequence, second red trial, third, etc.) is plotted in the $x$-axis. Baseline is the blocked condition for each observer. 
trial takes approximately $750 \mathrm{msec}$, and the intertrial interval is $2-2.5 \mathrm{sec}$. Thus, this short-term memory for the attention-focusing feature persists and can be maintained over a time period of approximately $30 \mathrm{sec}$ (but see the General Discussion for a qualification of this time effect).

\section{EXPERIMENT 8 \\ Mechanism: Target Facilitation or Distractor Inhibition}

We can think of two possible mechanisms that could mediate the priming of pop-out. First is target facilitationobservers may form a "search image" of the target with color repetition, thus facilitating the shift of attention to the odd color (Reid \& Shettleworth, 1992). Second is distractor inhibition-if distractors are more easily ignored by an attention-focusing mechanism, then attention will be more efficiently drawn to the odd-colored target.

In order to examine each potential mechanism in isolation, we took advantage of the fact that the priming effect is cumulative (as described in Experiment 7). To test the target effect, we kept the color of the target the same over several trials and changed the distractor color on each trial, or, conversely, we kept the distractor color the same over a sequence of trials and changed the target color on each trial. For each condition, we measured the amount of cumulative priming over a short sequence. This allowed us to see whether there is an effect for target color that is separate from that for distractor color and, if there is, to compare the amount of priming for each.

\section{Method}

Subjects. Two practiced observers participated in this experiment.

Stimuli. Blue and yellow were used in this experiment, in addition to the red and green used previously. The four colors made 12 possible combinations of target and distractor colors. In order to have 12 equally discriminable combinations of colors, we changed the colors slightly. The luminance and CIE coordinates for the different color elements were as follows: red- $.524 / .286$, luminance $3.92 \mathrm{~cd} / \mathrm{m}^{2} ;$ green- $.296 / .580$, luminance $3.79 \mathrm{~cd} / \mathrm{m}^{2}$; yellow$.492 / .391$, luminance $4.72 \mathrm{~cd} / \mathrm{m}^{2}$; blue--.162/.103, luminance $2.08 \mathrm{~cd} / \mathrm{m}^{2}$. Other aspects of the stimuli were the same as those described in the General Method.

Procedure. Three different sequence lengths were used: 2, 4, and 6. These sequences came up randomly, as in Experiment 7. Within each sequence, one of two conditions was enforced: (1) target color was kept the same and distractor color was changed on every trial, or (2) distractor color was kept the same and target color was changed on every trial. In addition, once a sequence was over, the colors from the last trial of that sequence would alternate for a short sequence [(sequence length) $/ 2+1]$. The new sequence would then start with two colors other than the ones just seen. This was done to increase reaction times (color alternation slows down responding) and ensure that a new sequence would not be contaminated by the possible priming from the previous sequence.

Both of the observers participated in 1,800 trials each, in blocks of 200 .

\section{Results and Discussion}

The results are shown in Figure 12. Repetition of target color alone or distractor color alone both resulted in faster responding as the position in sequence was advanced.

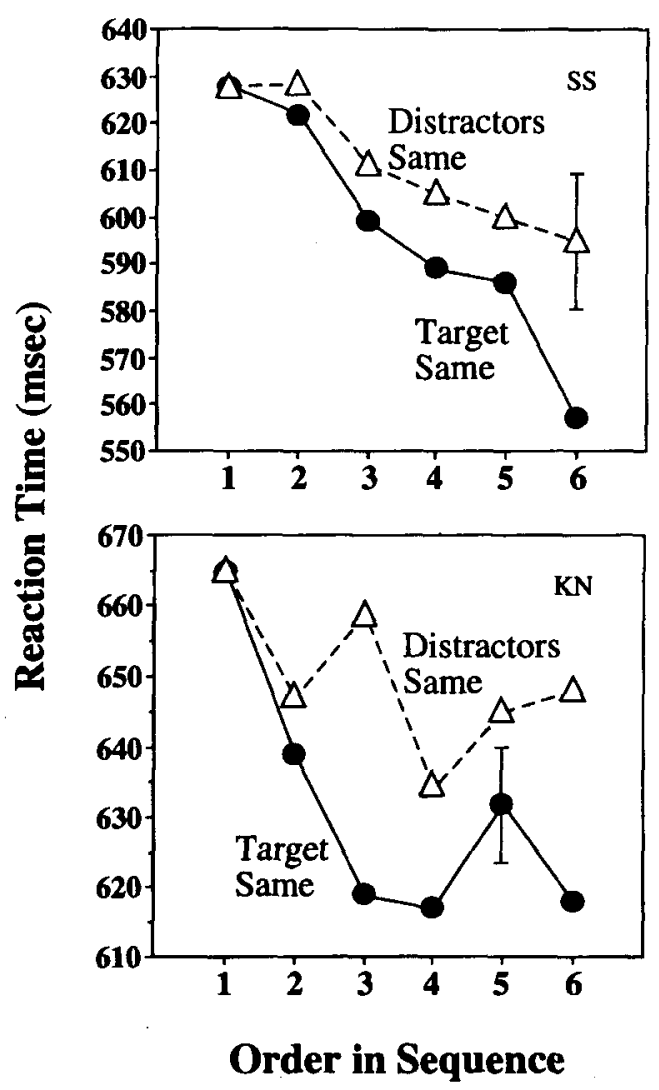

Figure 12. Experiment 8. Target facilitation as well as distractor inhibition mediate the priming effect. The filled circles show the decrease in reaction times when the target colors remained the same and the distractor colors varied over a short sequence. Open triangles show reaction times when the distractor color remained the same and the target color varied within a sequence.

Therefore, target facilitation and distractor inhibition both mediate the priming of pop-out, though the 2 observers showed greater summation when the target color was repeated than when the distractor color was repeated, suggesting that target facilitation was the more important mechanism.

\section{EXPERIMENT 9 Binocular Transfer}

To see how early this priming effect might be taking place, we used the binocular transfer technique. If an effect is eye specific, it probably takes place before the inputs from two eyes converge, at or before the primary visual cortex, V1. If the effect transfers, the effect takes place after the convergence of inputs. Some perceptual learning effects, such as discriminating the orientation of a line defined by texture (Karni \& Sagi, 1991) and performance on the vernier acuity task (Poggio, Fahle, \& Edelman, 1992), have been shown to be eye specific. We performed this experiment as a first attempt to localize the priming of pop-out at a neural level. 


\section{Method}

Subjects. One practiced observer, K.N., and a naive observer, P.V., participated in this experiment

Stimuli. The observers participated in the same "basic paradigm" that was used in the previous experiments. The colors were red and green, and there were always two distractors. In this experiment, however, we presented the three-element display to just one eye at a time. In order to do this, we used a mirror stereoscopic apparatus, which enabled us to present stimuli to one eye or to the other. We placed fixation points within two $7.5^{\circ} \times 7.5^{\circ}$ squares. The frames and the fixation points stayed on the screen throughout the experiment and were viewed binocularly, so that the observer saw a single frame with a fixation point in the center. The stimulus, which consisted of the target and two distractors, was presented within this binocularly fused square, and the elements were placed around an ellipse (major and minor axes were $5.2^{\circ}$ and $4.5^{\circ}$, respectively). The stimulus was presented to only one eye or the other on a random basis. Because the display was seen through the stereoscope, the observer did not know which eye was viewing the stimulus.

Procedure. The procedure that the observers followed was identical to that described in the General Method. From trial to trial, a stimulus was equally likely to be the same or a different color. In addition, the stimuli were presented to the left and right eyes on a random basis. Each observer participated in 520 trials.

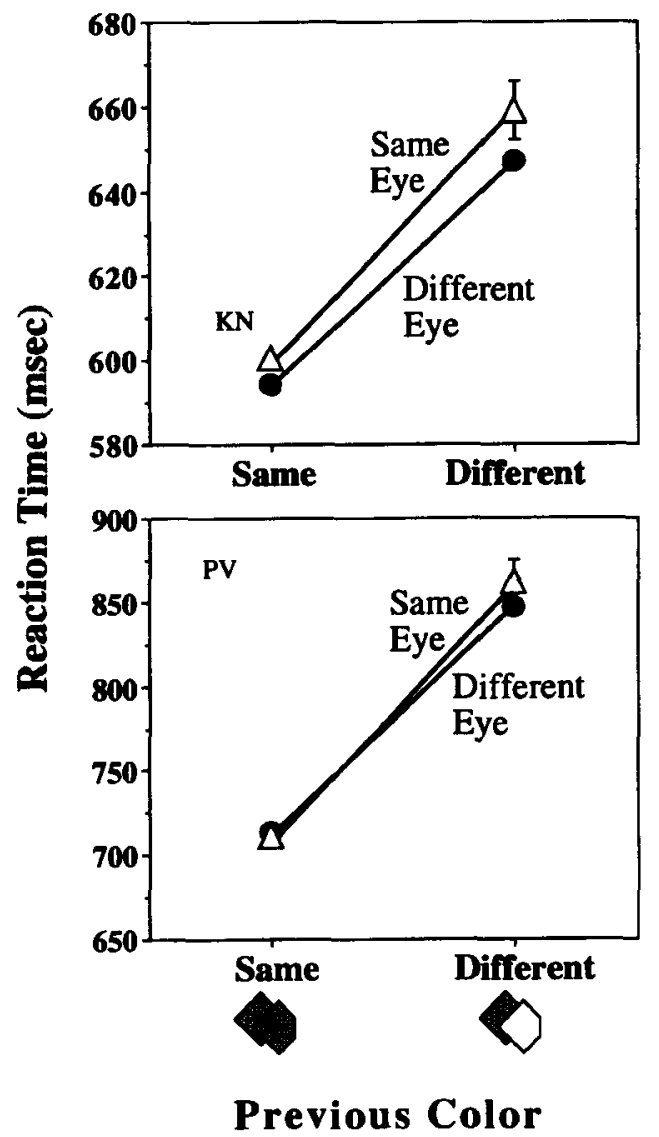

Figure 13. Experiment 9. Complete binocular transfer. Shown is a comparison of reaction times for same and different colors with respect to whether the array was presented to the same eye or a different eye. There is a clear same-color superiority, which is identical for the two conditions.

\section{Results and Discussion}

Figure 13 contains the data for both observers. There was no interaction between previous eye and previous color.

Both of the observers were faster after presentation of same-color stimuli than they were after different-color stimuli, and this reaction time difference was just as great when the stimulus was viewed with the same or a different eye. We conclude that pop-out priming by the previous stimulus color occurs after the inputs from two eyes have converged, either at or after primary visual cortex.

\section{GENERAL DISCUSSION}

\section{Relation to Previous Studies of Priming}

Our data suggest that the formation of the memory trace as well as its effect on subsequent trials are passive, autonomous processes. First, the task requires no explicit knowledge of the previous trial, yet its influence is substantial. Moreover, there is no established memory for the feature that one responds to directly (shape), but there is memory for the feature that is responded to indirectly (color, spatial frequency). Second, once the memory is established, it facilitates consecutive samecolor trials in a passive way-observers' knowledge or an attempt to apply it have no effect. These characteristics are quite similar to those obtained in implicit memory studies (for a review, see Schacter, 1990), suggesting that the priming of pop-out is a consequence of a short-term implicit memory for the attention-focusing feature. Preliminary studies directed specifically at this question support this implicit memory hypothesis (Maljkovic \& Nakayama, 1993).

Although the priming of pop-out satisfies the criteria for being an example of implicit memory, these criteria have been established by studies that differ significantly from the present study, both in the questions asked and in the methods used. We briefly review relevant studies to clarify the differences.

Our knowledge of the perceptual implicit memory comes mainly from the studies of "repetition priming." Repetition priming is a phenomenon in which studied items are more likely to be identified or are identified faster than items that had not been studied. A typical paradigm used in these studies is a "study-test" paradigm (Schacter, Delaney, \& Merikle, 1990) - a series of items is first presented to observers for study, then some of them are repeated among new items and identification is required. The studied items are identified faster or more easily.

The memory that underlies this priming is considered implicit. It is distinct from explicit memory, because the observers' ability to recall the episode in which a particular piece of knowledge has been acquired does not affect the priming results.

Priming occurs for familiar and novel objects. Researchers who have used priming of familiar objects are Warren and Morton (1982), who found that observers 
required less exposure to previously seen line drawings in order to name them, and Parkin and Streete (1988), whose observers were able to recognize a repeated object when it was shown in a more degraded form than an object not previously seen. In the first study, priming was observed $45 \mathrm{~min}$ later, and in the second study it was seen 2 weeks later. Mitchell and Brown (1988) found naming of previously seen objects to be faster than naming of new ones as long as 6 weeks after the initial study phase.

Perceptual priming in these studies appears to be quite specific-greatest facilitation appears to occur when visually identical stimuli are repeated. This finding, together with the finding that priming exists for novel objects (Musen \& Treisman, 1990; Schacter, Cooper, \& Delaney, 1990), led Schacter (1990) to posit the existence of "perceptual representation systems," which are dedicated to processing information about the specific visual structure and form of objects. As such, these subsystems process specific new representations-not abstract old representations - of objects.

The priming we observe may be a result of such a perceptual representation system function because an important aspect of the visual stimulus gets primed, but it is different from the findings described above because it is limited to the attention-focusing feature, and it is apparently absent for the representation of the shape per se.

The other aspect in which our finding differs is memory persistence. Other priming effects last from $45 \mathrm{~min}$ to several weeks, but the memory of the attentionfocusing feature lasts for only about five to eight trials, or about $30 \mathrm{sec}$ with our intertrial interval. We note, however, that the effects of trial number and time are completely confounded. In all but the first experiment, the intertrial interval was $2-2.5 \mathrm{sec}$. We know that the priming effect lasts for five to eight trials, but we do not know whether it decays with the number of trials or with time. Moreover, in any given session, there are a considerable number of color switches, providing for some possible combination of proactive and retroactive inhibition. Each could cause unlearning in subsequent or previous items, respectively (Baddeley, 1986; Wickens, Born, \& Allen, 1963). It is possible that if we did not repeat a color over a number of trials, or if our intertrial intervals were considerably longer, the memory for color would be present over a longer period of time. Thus, it is of great interest to determine whether the dramatic difference in persistence reflects a distinct, implicit form of short-term memory.

A related priming phenomenon is the so-called "negative priming"-a mechanism that underlies attentional selection. Tipper (1985) found that when observers were given two overlapping drawings of common objects, one of which was to be named, the object that had to be ignored on the previous trial was named more slowly as the target on the current trial. Tipper, Weaver, Cameron, Brehaut, and Bastedo (1991) further showed that this inhibition lasts for at least $7 \mathrm{sec}$, and it is not changed when predictable, unrelated (geometric shapes), inter- vening stimuli are inserted. Treisman and DeSchepper (1993) extended these findings to show that negative priming exists for novel shapes as well, that it saturates with a single repetition, and that it can last for over 100 intervening trials.

This negative priming effect is formally similar to the effects of previous distractor color and previous distractor position in our task (the distractor-position effect will be detailed in our next paper). The mechanism that underlies priming of pop-out relies on facilitation of target color as well as inhibition of distractor color. However, the stimulus dimensions we used were much more primitive (color, spatial frequency) and the task itself was different. The odd feature had to be detected first, and then discrimination was done on the object itself. The priming we describe is also cumulative over five to eight trials, and it does not saturate with a single exposure.

Given that our phenomenon shows priming for very simple stimulus dimensions, we also mention other, possibly related phenomena that are not related to popout - that is, priming in situations in which attention does not become spatially focused.

There are several studies that suggest that a shortlasting visual memory may be relevant in visual perception. In a study by Epstein and Rock (1960), observers named two pictures. They either saw one picture four times in a row, or saw one picture three times and then the other picture once. The observers knew whether the last picture would be the same or different and they were familiar with both, yet they performed better when the picture was always the same. In a study of motion detection, Sekuler and Ball (1977) found that thresholds were lower when motion direction was always the same within a block of trials rather than when trials were mixed. In simple present versus absent visual search tasks, both Treisman (1988), and Bravo and Nakayama (1992) showed very small but significant reductions in reaction times for blocked trials when compared with mixed trials. Finally, Robertson and Egly (1993) recently found that normal observers were better at allocating attention to global and local levels of hierarchically formed stimuli when spatial frequency stayed the same across successive trials.

A short-term priming mechanism akin to ours would account for these findings, although these data have not been subject to a trial-by-trial analysis. A practical issue is that the effects are very small; consequently, good sequential effects might be difficult to obtain. Nevertheless, such short-term priming may exist, and it could be a fairly general characteristic of perception that is not confined to the priming of pop-out.

\section{Related Literature on Sequential Trial Interactions}

Studies on sequential effects have shown two different and opposite findings. First are the "repetition effects," or facilitation when stimuli repeat. These are generally found with an intertrial interval shorter than $500 \mathrm{msec}$. Second are the "alternation effects"-f faster responding occurs with changing stimuli. These occur 
with intertrial intervals longer than $500 \mathrm{msec}$ (e.g., Kirby, 1972; Moss, Engel, \& Faberman, 1967). Although our finding may appear to contradict the results of these studies (we found a repetition effect with an intertrial interval that was significantly longer than $500 \mathrm{msec}$ ), we would like to point out that we have dissociated color, position, and shape/response, whereas these variables were confounded in the previously mentioned studies. Thus, our study of the priming of the attention-driving feature (color, spatial frequency) was uncontaminated by processes related to shape, position, or response.

Priming may also account for the data obtained in two-choice reaction time paradigms (e.g., Geller, Whitman, Wrenn, \& Shipley, 1971; Kirby, 1972; Remington, 1969; Schvaneveldt \& Chase, 1969; see also Kowler, Martins, \& Pavel, 1984), which show an orderly treelike arrangement. In these paradigms, each repetition of the stimulus produces a faster response, and alternation produces a slower response (indeed, our data produced the same kind of an orderly "tree" for the attentionfocusing feature when we analyzed all possible fifthorder sequences).

\section{Possible Functional Role of the Priming of Pop-Out}

In speculating on a possible functional role for the short-term-memory system that we have uncovered, we have been influenced by the very surprising results of Ballard, Hayhoe, Li, and Whitehead (1993), who monitored performance in a copying task. In their recently reported experiment, subjects were required to duplicate an arrangement of colored chips at an adjacent area, picking up colored chips from an available pile. What was surprising is that, rather than taking one look at the model area, memorizing its position and color, and then placing an available chip in the new copy area, their observers appeared to continually check back and forth from the model to the copy area. Rather than store the amount of information to choose and move a single chip, it appeared that they stored only the tiniest amount of information required for the next subcomponent of motor behavior-not sufficient for the whole sequence. They then executed this behavior, again picked up the minimal information for the next subcomponent, and so on. If this task is at all representative of things we do in our daily lives, then it would seem that rapid and efficient reorienting of our gaze direction would be critical for "chaining" complex sequences of motor behavior. The priming of pop-out might serve just such a role. Fischer (1987) postulated that there is an obligatory relation between eye movements and attentional shifts, in that eye movements cannot be made without an attentional shift (see also Mackeben \& Nakayama, 1993). Preliminary experiments recording eye movements in a variant of our particular task (Skavenski, McPeek, Maljkovic, \& Nakayama, 1993) support this view. We show that saccadic latencies to same-color targets become progressively shorter as that same color is repeated. The short-term memory then may facilitate this moment-to-moment orientation related to motor actions of the body.

\section{SUMMARY}

We have shown in this paper that pop-out can be primed. In order to do so, we used a task that disambiguated the attention-focusing feature of the task (odd color, odd spatial frequency) from the feature that required attention (shape, vernier offset). Manipulating the contingencies of the attention-focusing feature allowed us to show that observers' expectancies of an upcoming feature cannot affect their deployment of visual attention. Priming of pop-out is an autonomous process that is not influenced by conscious effort, and it is due to a short-term implicit memory of the attention-focusing feature.

The priming takes place over a time period of approximately $30 \mathrm{sec}$. We have shown that, over this time, one can track the influence of a single trial. The influence is the strongest immediately after a given current trial and then weakens gradually over the following five to eight trials. The effect of these 5-8 trials is cumulative, leading to a great improvement in performance when stimuli are repeated compared to when they alternate. As such, it accounts for the difference in reaction times for the "known" and "unknown" condition reported by Bravo and Nakayama (1992).

The priming of pop-out is mediated by memory for target color as well as for distractor color; target color has a greater facilitation effect, which takes place after the inputs from two eyes are combined. Presentation of the stimuli in the same hemifield does not produce any additional facilitation, suggesting that the pop-out priming may take place in an area where spatiotopic information about the stimulus is lost (we describe position priming effects in an upcoming paper).

\section{REFERENCES}

ATTNEAVE, F. (1959). Application of information theory to psychology: A summary of basic concepts, methods, and results. New York: Holt.

BADDEley, A. (1986). Working memory. New York: Oxford University Press.

Ballard, D., Hayhoe, M. M., Li, F., \& Whitehead, S. D. (1992). Hand-eye coordination during sequential tasks. Philosophical Transactions of the Royal Society of London: Series B, 337, 331-339.

Bravo, M. J., \& Nakayama, K. (1992). The role of attention in different visual-search tasks. Perception \& Psychophysics, 51, 465-472.

Cohn, T. E., \& WardLaW, J. C. (1985). Effect of large spatial uncertainty on foveal luminance increment detectability. Journal of the Optical Society of America A, 2, 820-825.

Davis, E. T., \& Graham, N. (1981). Spatial frequency uncertainty effects in the detection of sinusoidal gratings. Vision Research, 21, 705-712.

DunCAN, J., \& HumphreYs, G. W. (1989). Visual search and stimulus similarity. Psychological Review, 96, 433-458.

EPSTEIN, W., \& Rock, I. (1960). Perceptual set as an artifact of recency. American Journal of Psychology, 73, 214-228.

FISCHER, B. (1987). The preparation of visually guided saccades. Review of Physiology, Biochemistry, \& Pharmacology, 106, 2-35. 
Geller, E. S., Whitman, C. P., Wrenn, R. F., \& Shipley, W. (1971). Probability learning and a negative recency effect in the serial anticipation of alternative symbols. Journal of Experimental Psychologv, 41, 291-297.

JARVIK, M. E. (1951). Probability learning and a negative recency effect in the serial anticipation of alternative symbols. Journal of Experimental Psychology, 41, 291-297.

JuLESZ, B. (1986). Texton gradients: The texton theory revisited. $B i$ ological Cybernetics, 54, 245-251.

KARNI, A., \& SAGI, D. (1991). Where practice makes perfect in texture discrimination: Evidence for primary visual cortex plasticity. Proceedings of the National Academy of Sciences, 88, 4966-4970.

KiRBY, N. H. (1972). Sequential effects in serial reaction time. Journal of Experimental Psychology, 96, 32-36.

Косн, C., \& Ullman, S. (1985). Shifts in selective visual attention: Towards the underlying neural circuitry. Human Neurobiology, 4, 219-227.

Kowler, E., Martins, A. J., \& Pavel, M. (1984). The effect of expectations on slow oculomotor control: IV. Anticipatory smooth eye movements depend on prior target motions. Vision Research, 24, 197-210.

Kröse, B. J. A., \& JULESZ, B. (1989). The control and speed of shifts of attention. Vision Research, 29, 1607-1619.

Mackeben, M., \& NaKaYAMa, K. (1993). Express attentional shifts. Vision Research, 33, 85-90.

Maljkovic, V., Bravo, M., \& Nakayama, K. (1992). Characteristics of short-term visual memory which passively influence the guidance of focal attention. Perception, 21(Suppl. 2), 68.

Maljkovic, V., \& Nakayama, K. (1993). Priming of popout: An example of implicit short-term memory. Society for Neuroscience Abstracts, 19, 439.

Mitchell, D. B., \& Brown, A. S. (1988). Persistent repetition priming in picture naming and its dissociation from recognition memory. Journal of Experimental Psychology: Learning, Memory, \& Cognition, 14, 213-222.

Moss, S. M., ENGEL, S., \& Faberman, D. (1967). Alternation and repetition reaction times under three schedules of even sequencing. Psychonomic Science, 9, 557-558.

Musen, G., \& TReisman, A. (1990). Implicit and explicit memory for visual patterns. Journal of Experimental Psychology: Learning, Memory, \& Cognition, 16, 127-137.

Parkin, A. J., \& STREete, S. (1988). Implicit and explicit memory in young children and adults. British Journal of Psychology, 79, 361-369.

Poggio, T., Fahle, M., \& Edelman, S. (1992). Fast perceptual learning in visual hyperacuity. Science, 256, 1018-1021.

REID, P. J., \& SHETTLEWORTh, S. J. (1992). Detection of cryptic prey: Search image or search rate? Journal of Experimental Psychology: Animal Behavior Processes, 18, 273-286.

Remington, R. J. (1969). Analysis of sequential effects in choice reaction times. Journal of Experimental Psychology, 82, 250-257.

Rizzolati, G., Riggio, L., Dascola, I., \& Umiltà, C. (1987). Reorienting attention across the horizontal and vertical meridians: Evidence in favour of a premotor theory of attention. Neuropsychologia, 25, 31-40.

Robertson, L. C., \& Egly, R. (1993, November). Attention, spatial frequency, and parietal function in humans. Paper presented at the meeting of the Psychonomic Society, Washington, DC.

Rovamo, J., \& VIRSU, V. (1979). Visual resolution, contrast sensitivity, and the cortical magnification factor. Experimental Brain Research, 37, 475-494.

SAGI, D., \& Julesz, B. (1985). "Where" and "what" in vision. Science, 228, $1217-1219$.

SCHACTER, D. L. (1990). Perceptual representation systems and implicit memory: Toward a resolution of the multiple memory debate. Annals of the New York Academy of Sciences, 608, 543-571.

Schacter, D. L., CoOper, L. A., \& Delaney, S. M. (1990). Implicit memory for unfamiliar objects depends on access to structural descriptions. Journal of Experimental Psychology: General, 119, 5-24.

Schacter, D. L., Delaney, S. M., \& Merikle, E. P. (1990). Priming of nonverbal information and the nature of implicit memory. The Psychology of Learning \& Motivation, 26, 83-123.

SCHNEIDER, W., \& SHIFFrin, R. M. (1977). Controlled and automatic human information processing: I. Detection, search, and attention. Psychological Review, 84, 1-66.

Schvaneveldt, R. W., \& Chase, W. G. (1969). Sequential effects in choice reaction time. Journal of Experimental Psychology, 80, 1-8.

SEKULER, R., \& BALL, K. (1977). Mental set alters visibility of moving targets. Science, 198, 60-62.

Skavenski, A. A., McPeek, R. M., Maljkovic, V., \& Nakayama, K. (1993). Two forms of shortened saccadic eye movement latency in a popout visual search task. Society for Neuroscience Abstracts, 19, 426.

TIPPER, S. P. (1985). The negative priming effect: Inhibitory priming by ignored objects. Quarterly Journal of Experimental Psychology, 37A, 571-590.

Tipper, S. P., Weaver, B., Cameron, S., Brehaut, J. C., \& Bastedo, J. (1991). Inhibitory mechanisms of attention in identification and localization tasks: Time course and disruption. Journal of Experimental Psychology: Learning, Memory, \& Cognition, 17, 681-692.

Treisman, A. (1988). Features and objects: The fourteenth Bartlett memorial lecture. Quarterly Journal of Experimental Psychology, 40A, 201-237.

Treisman, A., \& DeSchepper, B. (1993). Memory for novel visual shapes. Investigative Ophthalmology \& Visual Science (Suppl.), 34, 1288.

Treisman, A., \& Gelade, G. (1980). A feature-integration theory of attention. Cognitive Psychology, 12, 97-136.

WARREN, C., \& MORTON, J. (1982). The effects of priming on picture recognition. British Journal of Psychology, 73, 117-129.

Wickens, D. D., Born, D. G., \& Allen, C. K. (1963). Proactive inhibition and item similarity in short-term memory. Journal of Verbal Learning \& Verbal Behavior, 10, 382-385.

Wolfe, J. M., Cave, K. R., \& Franzel, S. L. (1989). Guided search: An alternative to the feature integration model for visual search. Journal of Experimental Psychology: Human Perception \& Performance, 15, 419-433.

WURTZ, R. H., GoldberG, M. E., \& Robinson, D. L. (1980). Behavioral modulation of visual responses in the monkey: Stimulus selection for attention and movement. Progress in Psychobiology \& Physiological Psychology, 9, 43-83.

\section{NOTES}

1. Our task requires relatively high acuity. Acuity falls off faster along the vertical axis than along the horizontal axis (Kröse \& Julesz, 1989; Rovamo \& Virsu, 1979). By using an ellipse, performance is equalized for all positions of the target.

2. The "blocked" and "mixed" conditions are similar to the "consistent mapping" and "variable mapping" conditions, respectively, of Schneider and Shiffrin (1977).

3. To change the probability of the color switch, we used the following procedure. A random number between 0 and 1 was chosen at every trial. If this number was below $p$ (switch), the colors of target and distractor colors would reverse on the upcoming trial. If the random number was higher than $p$ (switch), the target and distractor colors would stay the same.

4. Note that although we have disambiguated the effect of color by having the observers respond to it only indirectly, the shape and response are confounded. Consequently, we use "shape" and "response" intermittently or together.

5. The "active" and "passive" conditions are similar to the "fullattention" and "shared-attention" conditions, respectively. In the passive condition, Observer K.N. chatted with the author V.M.; Observer S.S. listened to the radio.

6 . In the pilot data that we collected for both color and spatialfrequency experiments, we found the shape/response data to be more variable than the data for the attention-driving features.

7. When two colors are equally likely to appear at every trial (i.e., in a Bernoulli sequence), longer and longer same-color sequences become more and more improbable, thußs providing little opportunity to examine the effects of many repetitions.

(Manuscript received April 15, 1993; revision accepted for publication November 29, 1993.) 